\title{
Fundamental flow measurement capabilities of optical Doppler and time-of-flight principles
}

\author{
Andreas Fischer ${ }^{1}$ (D) \\ Received: 15 September 2020 / Revised: 16 November 2020 / Accepted: 21 December 2020 / Published online: 30 January 2021 \\ (c) The Author(s) 2021
}

\begin{abstract}
In order to understand the fundamental measurement capabilities of different flow velocity measurement principles based on Mie scattering, a fundamental equation of how to calculate the shot noise limit for a respective signal model is derived. The derivation is based on the well-known rules of uncertainty propagation and yields the Cramér-Rao bound without the necessity to calculate the Fisher information. The derived equation is next applied to compare the shot noise limit for Doppler and time-of-flight principles including laser Doppler anemometry (LDA), planar Doppler velocimetry (PDV), laser-two-focus velocimetry (L2F), particle tracking velocimetry (PTV) and particle image velocimetry (PIV). The comparison is performed for an identical mean laser power, while two cases are studied in detail: measuring on a single seeding particle as well as measuring on multiple seeding particles and averaging. LDA, L2F and PTV/PIV obey a similar shot noise limit. For the case of a measurement on multiple seeding particles, the minimal achievable measurement uncertainty is directly proportional to the absolute value of the measured velocity component and inversely proportional to the spatial resolution. The respective shot noise limit for PDV is almost independent of the measured flow velocity component and the spatial resolution. Since PDV is sensitive with respect to a different flow velocity component depending on the observation direction, a comparison with the other principles is only reasonable to a certain extent. However, all shot noise limits in case of measuring on multiple seeding particles show the expected inverse proportionality to the square root of the total number of detected photons and thus also to the square root of the measurement time. Considering a comparable spatiotemporal resolution, an identical mean light power and typical measurement configurations, the PDV shot noise limit is the largest. As a final result, it is derived that each measurement principle obeys an uncertainty principle between position and the respective component of the wave vector, which is in agreement with Heisenberg's uncertainty principle. Therefore, a common basis is provided to assess the fundamental measurement capabilities of Doppler and time-of-flight measurement systems on the basis of what is possible within the quantum mechanical constraints.
\end{abstract}

Andreas Fischer

andreas.fischer@bimaq.de

1 Bremen Institute for Metrology, Automation and Quality Science (BIMAQ), University of Bremen, Linzer Str. 13, 28359 Bremen, Germany 


\section{Graphic abstract}

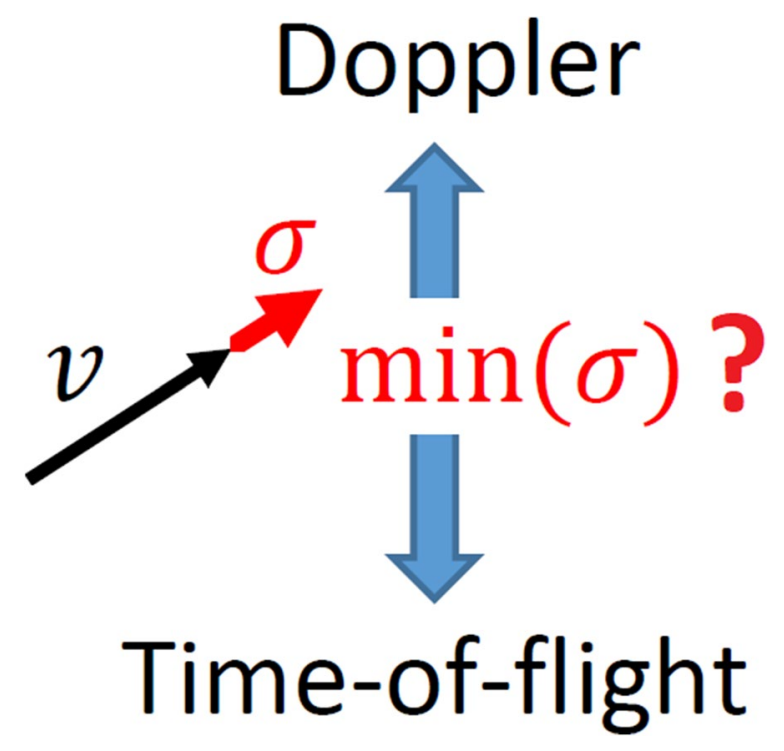

\section{Introduction}

\subsection{Motivation}

When confronted with a flow measurement task, finding the proper choice from the huge variety of different measurement principles is difficult. Various optical principles exist, which enable contactless and fast flow velocity measurements. Even hybrid optical measurement methods are known and were, for example, proposed by Grosjean et al. (1997), Förster et al. (2000), Wernet (2004) and Willert et al. (2006). However, regarding the capabilities of the different optical measurement methods, the majority of the studies (aside from some exceptions such as Willert et al. 2005; Thacker et al. 2010) deal only with a single measurement approach. Benchmark tests, in particular over more than two different measurement methods, are rare or even missing. Furthermore, a flow measurement system is typically rated or chosen from what is currently available considering technological and economical aspects. In order to understand the fundamental measurement capabilities of the various optical flow measurement principles-even with respect to future technological developments-and to identify the minimal achievable measurement uncertainty, a scientific study about the fundamental limits of measurability is required.

\subsection{State of the art}

Before reviewing the scientific studies regarding a physical lower limit of the measurement uncertainty, the measurement principles are introduced. The present article is focused on optical flow measurements based on laser light and Mie scattering, because these measurements provide the highest signal-to-noise ratio that for instance allows high-speed measurements even with $\mathrm{MHz}$ rate and imaging capability (Thurow et al. 2013). The so-called seeding particles are thus added to the fluid (if not naturally present), which are sufficiently small to follow the flow with negligible slip but at the same time sufficiently large to provide Mie scattering (Albrecht et al. 2003). The scattered light intensity for the Mie scattering on seeding particles is about 10 to 15 orders of magnitude larger than what can be achieved for Rayleigh scattering on the fluid molecules (Bohren and Huffman 2004). As a result, the particle velocity is actually measured and, by assuming a negligible slip, is then interpreted as the flow velocity. According to the review article (Fischer 2017a) from 2017, the flow velocity measurement principles using Mie scattering can by categorized into Doppler and time-of-flight principles. For each category, the developed measurement methods are subsequently summarized.

Doppler principles evaluate the frequency change of the scattered light from a particle that is caused by a nonzero flow velocity relative to the measurement system. The Doppler frequency is the light frequency difference between illumination and observation. One technique to measure the Doppler frequency directly is to mix the light from 
illumination and observation, since the intensity of the mixed light signal oscillates with the Doppler frequency. The latter is thus extracted from the detected intensity of the mixed signal using a frequency analysis. An early but still state-of-the-art measurement method that is based on the direct Doppler frequency measurement is Laser Doppler Anemometry (LDA) (Yeh and Cummins 1964). Originally introduced as a point measurement device, enhanced LDA techniques enabling profile (Czarske 2001) and field measurements (Coupland 2000; Voigt et al. 2008; Meier and Rösgen 2012) are also known today. Another Doppler technique is to separately measure the frequency of the illuminated light and the observed scattered light, and evaluating the frequency difference in a second step. The required light frequency measures are enabled using an optical filter whose spectral transmission has a steep edge that converts changes in light frequency to changes in transmitted light intensity. The latter is measurable with a photodetector or a camera. Respective Doppler measurement methods have been developed with atomic or molecular filters (Meyers 1995; Charrett et al. 2004; Fischer et al. 2007; Müller et al. 2007; Cadel and Lowe 2015) and with interferometric filters such as a Fabry-Pérot interferometer (Jackson and Paul 1970; Büttner et al. 2013), a Michelson interferometer (Smeets and George 1978; Seiler and Oertel 1983; Landolt and Rösgen 2009) and a Mach-Zehnder interferometer (Lu et al. 2009). These methods are mostly denominated as Planar Doppler Velocimetry (PDV) or Doppler Global Velocimetry (DGV), since planar measurements are easily obtained with a light sheet illumination and cameras. However, scanning or multiplexing techniques also enable volumetric PDV measurements (Fischer et al. 2014, 2015).

Time-of-flight principles directly apply the definition of velocity as the derivative of the position with respect to the time. Therefore, the change in particle position for a priori determined time steps is evaluated, or vice versa, the elapsed time when the particle moves over a priori-determined spatial distances. In both cases, position as well as time differences need to be measured, but the uncertainty of the a priori-determined quantity is usually negligibly small. Therefore, one distinguishes between time-of-flight methods with position measurements and time measurements. Note that the differential quotient of the position over time is approximated for most of the applications by the difference quotient. The measurement method that is based on two time measurements is Laser-2-Focus Anemometry (L2F) (Tanner 1973), where the particle subsequently crosses two parallel laser beams and the two particle arrival times are obtained from the detected scattered light pulses. The method is established for point measurements, but can in principle be enhanced toward profile and field measurements with an increased number of laser beam pairs and a camera. A more popular field measurement method that directly measures the time differences of predefined spatial distances is Spatial Filter Velocimetry (SFV) (Aizu and Asakura 1987). Instead of a spatially structured illumination, SFV uses a receiving aperture with a spatially structured sensitivity by applying an optical fringe-type absorption filter. Volumetric SFV measurements are also possible (Hosokawa et al. 2013). However, the time-of-flight measurement methods that are based on position measurements are currently the most popular methods for optical flow velocity field measurements. Particles in a laser light sheet are imaged with a camera for two short laser pulses with a predefined pulse separation time. The position change of each single particle or a pattern of multiple particle images is then obtained by the cross-correlation of sub-images. The method of tracking single particles is Particle Tracking Velocimetry (PTV) (Adrian 1991; Maas et al. 1993). Tracking a pattern of multiple particle images in the interrogation window is Particle Imaging Velocimetry (PIV) (Adrian 2005). Several volumetric PTV/PIV measurement methods are known aside from scanning: for instance, by using tomography (Scarano 2013), holography (Hinsch 2002), light field technique (Cenedese et al. 2012; Fahringer et al. 2015) and synthetic apertures (Cierpka et al. 2010; Belden et al. 2010).

As a result, different Doppler and time-of-flight principles for flow velocity field measurements exist, which are in the following represented by LDA as Doppler principle using light mixing, PDV as Doppler principle using light filtering, L2F as time-of-flight principle based on time measurements and PTV/PIV as time-of-flight principle based on position measurements.

Regarding a physical lower limit of the measurement uncertainty, the error propagation from the naturally fluctuating number of scattered photons was studied for each measurement method. The effect of fluctuating number of photons is known as photon shot noise, which is a fundamental, ultimate and inevitable source of uncertainty. Considering non-squeezed monochromatic laser light, the number of scattered photons that occur in a certain time interval follows a Poisson distribution. Therefore, an uncertainty propagation calculation considering Poissonian noise was accomplished for LDA and L2F by Oliver (1980) in 1980, and Lading and Edwards (1993) studied the extraction of the velocity information from LDA and L2F signals with noise in 1993. McKenzie (1996) investigated the PDV measurement uncertainty in 1996 and included an uncertainty propagation calculation for Poissonian noise. Using an information theoretic approach, the Cramér-Rao bound $(C R B)$ was further calculated for all variants of PDV with and without laser frequency modulation by Fischer (2017) in 2017. The Cramér-Rao bound is obtained from the inverse of the Fisher information and equals the minimal achievable measurement uncertainty squared for any unbiased estimator. The same information theoretic approach was used to 
derive the lower uncertainty limit for PTV by Wernet and Pline (1993) in 1993. Note that the PTV solution is also applicable for PIV when the particle images do not overlap. The measurement uncertainty then reduces inversely proportional to the square root of the number of particle images in the interrogation window. Furthermore, a first attempt to compare the photon shot noise limits of LDA, PDV and L2F using a uniform information theoretic approach took place in 2010 (Fischer et al. 2010) and was briefly complemented with PTV in 2017 (Fischer 2017a). As a result, the minimal achievable measurement uncertainty for LDA, L2F and PTV is similar while it is comparably high for PDV because the scattered light and the information obtained is attenuated by the light filtering. However, the relation between the applied Fisher information calculation (approach by Rao 1945 and Cramér 1946, see also Casella and Berger 1990) and a classical uncertainty propagation calculation using linear approximation (approach by C. F. Gauß that is included in the international guide to the expression of uncertainty in measurement Joint Committee for Guides in Metrology 2008) was not addressed. In particular, it is worth studying under which circumstances an uncertainty propagation calculation equals the same flow velocity uncertainty limit as the more cumbersome calculation of the Fisher information and the Cramér-Rao bound.

Furthermore, the existing comparative studies do not focus on the dependency of the shot noise limit on the spatiotemporal resolution. This dependency is important to assess the fundamental measurement capabilities.

Since photons follow the natural laws of quantum mechanics, Heisenberg's uncertainty principle (Heisenberg 1927) is another approach to calculate the fundamental limit of measurement uncertainty due to photon shot noise. This physical approach by W. Heisenberg leads to the same results as with Poissonian photon distribution and information theory (Teich and Saleh 1989), which was explicitly proven for LDA and L2F (Fischer 2016). The surprise of this comparative study was that aside from proportionality factors, an equal limit for the flow velocity measurement uncertainty is obtained for L2F and LDA. This seems remarkable because LDA as Doppler principle evaluates the momentum information of the scattered photons while L2F as time-of-flight principle evaluates the position information of the scattered light photons, so from the physical perspective two completely different, complementary measurement principles. A broader respective study of Heisenberg's uncertainty principle that additionally covers PDV and PTV/PIV is an open task. However, the results from Heisenberg's uncertainty principle will be identical with the consideration of a Poissonian photon distribution. The interesting statement of Heisenberg's uncertainty principle is that the product of the position uncertainty and the momentum uncertainty of a quantum mechanical object (such as the photon) cannot be reduced below a certain quantum limit. A reduced position measurement uncertainty is thus only enabled by an increased momentum uncertainty and vice versa. This quantum mechanical perspective initiated a uniform physical understanding and review of the various optical position and shape measurement methods (Pavliček and Häusler 2014; Pavliček and Pech 2016; Fischer 2019), but the aspect of a fundamental uncertainty relation that enables the minimization of the flow velocity measurement uncertainty at the cost of a maximized uncertainty of a complementary quantity has not yet been fully considered in a comparative study of LDA, PDV, L2F and PTV/PIV.

\subsection{Aim and structure of the article}

The first aim of the article is to replace the calculation of the Cramér-Rao bound by the more common and simple rules of uncertainty propagation and to derive the photon shot noise limits for LDA, PDV, L2F and PTV/PIV. The second aim is to compare the derived fundamental measurement limits and to understand the respective dependency on the spatiotemporal resolution. The third aim is to understand the uncertainty relations for the optical flow velocity measurements based on laser Mie scattering, i.e., what uncertainty has to be maximized to minimize the flow velocity measurement uncertainty?

The considered measurement methods, which represent Doppler principles with light mixing and light filtering as well as time-of-flight principles with position and time measurements, respectively, are described in Sect. 2. In the description of the principles, the comparability of the different measurement methods is addressed, i.e., fundamentally different measurement features are emphasized that indicate the uniqueness of each measurement principle. As a result, signal models for LDA, PDV, L2F and PTV/PIV are derived that are applicable to an uncertainty propagation calculation. The uncertainty propagation calculation that yields the minimal achievable measurement uncertainty for photon shot noise is presented in Sect. 3 . The simplified consideration of a single measurand as the only unknown quantity allows to apply the uncertainty propagation calculation in order to obtain the square root of the Cramér-Rao bound as the minimal achievable measurement uncertainty. This finding is applied to calculate the shot noise limits of LDA, PDV, L2F and PTV/PIV in Sect. 4. In addition, uncertainty relations are identified in the results and compared between the different measurement methods. The conclusions are drawn in Sect. 5 together with a discussion concerning remaining research questions. 


\section{Measurement principles}

A physical signal model for each measurement principle is required to calculate the respective shot noise limit. The signal modeling is based on several assumptions. Particle slip is not part of the following investigation, so that the actually measured particle velocity can be assumed to equal the flow velocity. Furthermore, the flow velocity components are considered as constant during the measurement time, because flow acceleration effects on the measurement result are not studied here. Flow turbulence is also excluded here, i.e., all particles that occur in the measurement volume have an identical velocity. For the sake of simplicity, monodisperse particles and a flow velocity vector with no out-of-plane component are further considered. Out-of-plane means out of the measurement plane, which is defined by the illumination. In order to focus on the fundamental physics of each measurement principle, technical aspects regarding the photo detection unit (photo detector or camera) and the signal digitization are not included in the signal modeling. In particular, the discretization of the detected light signal (or image) over time (due to sampling) and over space (due to camera pixels) is not part of the present study. Finally, the signal processing algorithm and its efficiency to precisely estimate the flow velocity are not discussed, because the article is focused on the comparison of different measurement principles using the Cramér-Rao bound. The Cramér-Rao bound for any unbiased estimator is independent from the signal processing algorithm.

\subsection{LDA}

A state-of-the-art differential LDA setup is considered as depicted in Fig. 1. The original laser beam with the wavelength $\lambda$ is split into two beams, which are here modeled as symmetric Gaussian beams with the $1 / e^{2}$-radius $2 w_{\text {beam }}$. Both beams are brought to intersection with the crossing angle $2 \alpha$. The intersection region is the measurement volume, in which an interference fringe system occurs that is observed with a photodetector. The fringe distance is

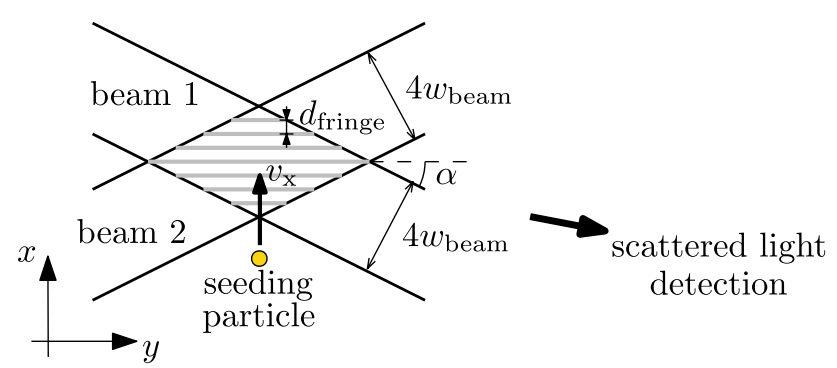

Fig. 1 LDA measurement principle $d_{\text {fringe }}=\frac{\lambda}{2 \sin \alpha}$. Due to the interference fringe system, the intensity distribution is a cosine-type modulation with a Gaussian envelope. Considering one seeding particle that crosses the fringe system at the arrival time $t_{0}$, the detected scattered light signal over time $t$ in unit number of photons (accumulated over one sampling period $\Delta t$ ) reads

$s_{t}\left(f_{\mathrm{D}}\right)=\frac{A_{\mathrm{LDA}} \cdot \Delta t}{\sqrt{2 \pi} \frac{w_{\text {fringes }}}{\left|v_{\mathrm{x}}\right|}} \exp \left(-\frac{\left(t-t_{0}\right)^{2}}{2 \cdot\left(\frac{w_{\text {fringes }}}{v_{\mathrm{x}}}\right)^{2}}\right) \cdot\left(1+\cos \left(2 \pi f_{\mathrm{D}} \cdot\left(t-t_{0}\right)\right)\right)$,

with

$A_{\mathrm{LDA}}=\dot{N}_{\text {photon, } \mathrm{LDA}} \cdot T_{\mathrm{LDA}} \cdot$

The amplitude $A_{\mathrm{LDA}}$ is the total number of detected photons, which is here defined by a mean photon rate $\dot{N}_{\text {photon,LDA }}$ multiplied by the transit time

$T_{\mathrm{LDA}}=\frac{4 w_{\text {fringes }}}{\left|v_{\mathrm{x}}\right|}$,

where $4 w_{\text {fringes }}=4 w_{\text {beam }} / \cos \alpha$ is the $\left(1 / e^{2}\right)$-extension of the measurement volume in $x$-direction and $v_{\mathrm{x}}$ denotes the respective flow velocity component. The temporal width of the Gaussian envelope is thus described by the lateral width of the beam intersection region divided by the flow velocity. The beat frequency of the signal due to the Doppler effect is the Doppler frequency $f_{\mathrm{D}}$. This LDA signal model is shown in Fig. 2.

The signal from Eq. (1) is evaluated for instance using harmonic analysis to estimate the Doppler frequency. Together with the previously determined fringe distance $d_{\text {fringe }}$ during calibration, the flow velocity component perpendicular to the fringes is finally obtained by calculating

$v_{\mathrm{x}}=f_{\mathrm{D}} \cdot d_{\text {fringe }} \cdot$

Sensitivity: The basic LDA measurement configuration yields one flow velocity component at a single point. The

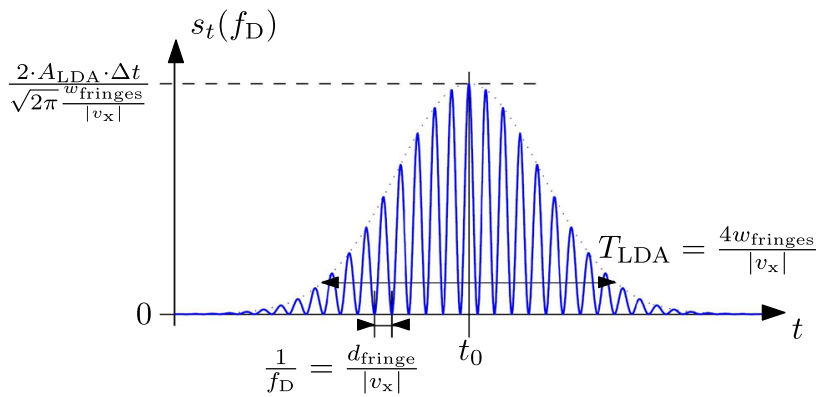

Fig. 2 LDA signal model 
sensitivity direction is perpendicular to fringes, which is in the same plane as the two laser beams and perpendicular to the optical axis of the illumination. The observation direction has no effect on the sensitivity direction.

Spatial resolution: The spatial resolution in all three dimensions is typically determined by the spatial extension of the fringe system (illumination) but might be additionally limited by the optical imaging on the photodetection unit (observation).

Temporal resolution: The temporal resolution for a single particle amounts to $T_{\mathrm{LDA}}$. It depends on the flow velocity and the width of the laser beams as well as the beam crossing angle. Note that varying the laser beam width or the beam crossing angle will affect the temporal as well as the spatial resolution. For a given measurement time $T \gg T_{\mathrm{LDA}}$ that allows to observe multiple particles crossing the fringe system, an averaging over floor $\left(T / T_{\mathrm{LDA}}\right)$ consecutive single particle measurements is considered here as the theoretic maximum

\subsection{PDV}

The flow is continuously illuminated with a laser light sheet which represents the measurement plane. Illumination intensity variations in the observed region of interest in the measurement plane are neglected here. The laser has a narrow bandwidth and has a stabilized center frequency $f_{\mathrm{c}}=c / \lambda$ according to the laser wavelength $\lambda$ and the light speed $c$. To detect the flow velocity-dependent frequency change of the light scattered on seeding particles, an imaging system including a beam splitter (here $50 \% / 50 \%$ ) and two digital cameras as well as a narrow-band spectral filter is applied, see Fig. 3. Half of the scattered photons from the illuminated seeding particles in the measurement plane are imaged with the reference camera. The other half of the scattered photons pass a spectral filter with the spectral

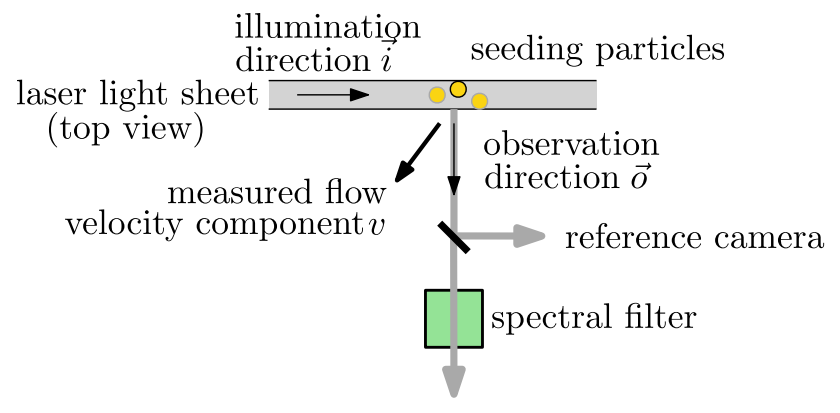

signal camera

Fig. 3 PDV measurement principle, where the laser light sheet is shown in top view. Note that the sensitivity vector is $\vec{o}-\vec{i}$. The observation direction is shown perpendicular to the light sheet plane $(\vec{i} \perp \vec{o})$, which is a typical configuration transmission $\tau\left(f_{\mathrm{c}}+f_{\mathrm{D}}\right)$, and the transmitted photons are imaged with the signal camera. The symbol $f_{\mathrm{D}}$ denotes the Doppler frequency shift. Note that the filter bandwidth is significantly larger than the bandwidth of the continuouswave laser, i.e., the illuminating laser can be considered as an ideal laser source with single frequency. As a result, the signals from the signal camera and the reference camera over time $t$ in unit number of photons (accumulated over one sampling period $\Delta t$ ) in the corresponding interrogation window (single pixel or superpixel) read for the presence of $N_{\text {particle,PDV }}$ seeding particles

$s_{t}\left(f_{\mathrm{D}}\right)=\frac{1}{2} \cdot N_{\mathrm{particle}, \mathrm{PDV}} \cdot A_{\mathrm{PDV}} \cdot \frac{\Delta t}{T_{\mathrm{PDV}}} \cdot \tau\left(f_{\mathrm{c}}+f_{\mathrm{D}}\right)$,

$s_{t, \text { ref }}\left(f_{\mathrm{D}}\right)=\frac{1}{2} \cdot N_{\text {particle, } \mathrm{PDV}} \cdot A_{\mathrm{PDV}} \cdot \frac{\Delta t}{T_{\mathrm{PDV}}}$,

with $A_{\mathrm{PDV}}$ as the total number of scattered photons per seeding particle during the particle transit time $T_{\mathrm{PDV}}$ through the interrogation window. Note that the number of observed seeding particles increases with an increasing size of the interrogation window and an increasing light sheet thickness. The mean photon rate $\dot{N}_{\text {photon,PDV }}$ per seeding particle is defined by the relation

$A_{\mathrm{PDV}}=\dot{N}_{\text {photon,PDV }} \cdot T_{\mathrm{PDV}} \cdot$

with

$T_{\mathrm{PDV}}=\frac{w_{\text {transit }}}{|v|}$.

This PDV signal model is shown in Fig. 4. The symbol $w_{\text {transit }}$ denotes the width of the illuminated interrogation region with respect to the PDV sensitivity direction $\frac{\vec{o}-\vec{i}}{|\vec{o}-\vec{i}|}$, and $v$ denotes the measured velocity component along the PDV sensitivity direction.

The Doppler frequency $f_{\mathrm{D}}$ is determined by evaluating the ratio $s_{t}\left(f_{\mathrm{D}}\right) / s_{t, \text { ref }}\left(f_{\mathrm{D}}\right)=\tau\left(f_{\mathrm{c}}+f_{\mathrm{D}}\right)$, by subtracting the laser working point $\tau\left(f_{\mathrm{c}}\right)$ and by applying the inverse transmission function that was previously calibrated. The flow

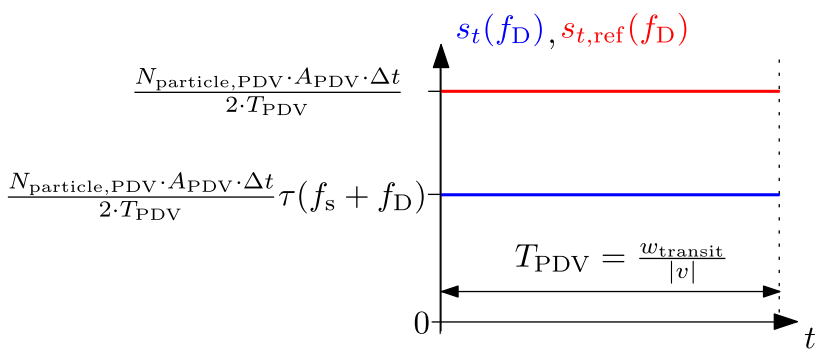

Fig. 4 PDV signal model 
velocity component $v$ along the sensitivity direction $\frac{\vec{o}-\vec{i}}{|\vec{o}-\vec{i}|}$ is then calculated according to the relation (Fischer 2017)

$v=\frac{\lambda}{|\vec{o}-\vec{i}|} \cdot f_{\mathrm{D}}$.

The unit vectors $\vec{i}$ and $\vec{o}$ describe the direction of illumination and observation, respectively.

Note that only a classical PDV without laser frequency modulation is considered here, since it was already shown in (Fischer 2017) that all variants of PDV with laser frequency modulation and without laser frequency modulation have similar measurement capabilities with respect to the shot noise limit.

Sensitivity: The PDV principle provides sensitivity with respect to the out-of-plane velocity component. The direction of sensitivity is $\frac{\vec{o}-\vec{i}}{|\vec{o}-\vec{i}|}$ and thus depends on the illumination direction and the observation direction. For a typical perpendicular arrangement of the light sheet plane and the observation, a mixture of in-plane and out-of-plane velocity component is measured. The in-plane velocity component along the light sheet propagation direction is measurable independent of the observation direction when two consecutive Doppler frequency measurements are performed with opposite illumination directions and the same observation direction (Roehle and Willert 2001). Due to the use of cameras, imaging capability is provided.

Spatial resolution: The out-of-plane spatial resolution is usually determined by the illumination, i.e., the light sheet thickness. This holds in particular for an observation direction perpendicular to the light sheet plane, which is a typical measurement configuration. The in-plane spatial resolutions are then determined by the chosen region of interest in the captured frame, which is either a single pixel or an area of binned pixels. A perpendicular arrangement between the light sheet plane and the observation direction allows the highest spatial resolution.

Temporal resolution: Note that multiple particles are allowed to be present in the interrogation window of the illuminated region and are measured simultaneously. The PDV measurement is possible even for overlapping particle images so that very high seeding particle concentrations can be applied. The temporal resolution is thus typically determined and adjustable by the camera exposure time $T$. Considering $T_{\mathrm{PDV}}$ as the time for a single measurement and $T>T_{\mathrm{PDV}}$, an averaging over $\left(T / T_{\mathrm{PDV}}\right)$ measurements takes place implicitly.

\section{$2.3 \mathrm{~L} 2 \mathrm{~F}$}

The flow is illuminated by two parallel laser beams, see Fig. 5. A photodetector is applied to detect the scattered

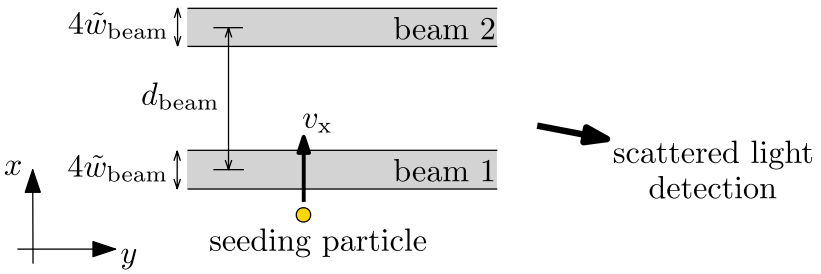

Fig. 5 L2F measurement principle

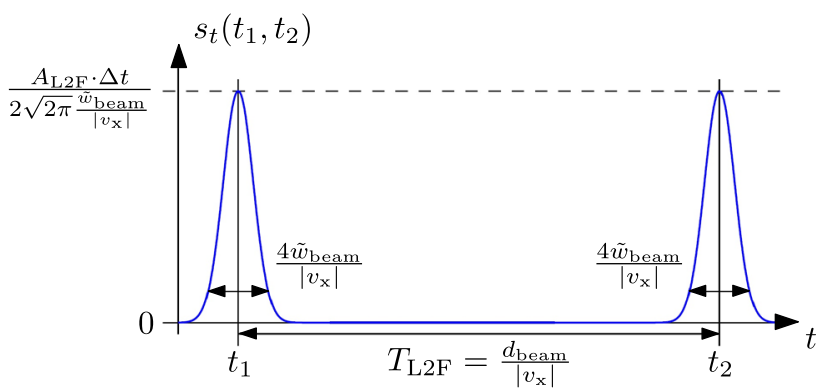

Fig. 6 L2F signal model

light from a seeding particle that crosses both laser beams in the region of interest. Assuming identical Gaussian beam intensity profiles, the sensor signal over the time $t$ contains two identical Gaussian pulses at time $t_{1}$ and $t_{2}$ and reads in unit number of photons (accumulated over the sampling period $\Delta t$ )

$s_{t}\left(t_{1}, t_{2}\right)=\sum_{i=1}^{2} \frac{1}{2} \cdot \frac{A_{\mathrm{L} 2 \mathrm{~F}} \cdot \Delta t}{\sqrt{2 \pi} \frac{\tilde{w}_{\text {beam }}}{\left|v_{\mathrm{x}}\right|}} \exp \left(-\frac{\left(t-t_{i}\right)^{2}}{2 \cdot\left(\frac{\tilde{w}_{\text {beam }}}{v_{\mathrm{x}}}\right)^{2}}\right)$

with

$A_{\mathrm{L} 2 \mathrm{~F}}=\dot{N}_{\text {photon,L2F }} \cdot T_{\mathrm{L} 2 \mathrm{~F}}$

The temporal width of the pulses as well as the energy content of the pulses scale with the inverse of the soughtafter velocity component $v_{\mathrm{x}}$. Therefore, the total number of detected photons $A_{\mathrm{L} 2 \mathrm{~F}}$ is written as a mean photon rate $\dot{N}_{\text {photon,L2F }}$ multiplied by the transit time

$T_{\mathrm{L} 2 \mathrm{~F}}=\frac{d_{\text {beam }}}{\left|v_{\mathrm{x}}\right|}$,

where $d_{\text {beam }}$ is the distance of the two laser beams. The temporal pulse half-width is $\left(2 \tilde{w}_{\text {beam }} /\left|v_{\mathrm{x}}\right|\right)$, where $2 \tilde{w}_{\text {beam }}$ denotes the $1 / e^{2}$-radius of each laser beam. This L2F signal model is shown in Fig. 6.

Since the overlap of the pulses is negligible, the sensor signal to be solved for the two parameters $t_{1}, t_{2}$ can also be 
written in the form of a combination of two signals $i=1,2$ with only one parameter $t_{i}$ each:

$s_{t, i}\left(t_{i}\right)=\frac{1}{2} \cdot \frac{A_{\mathrm{L} 2 \mathrm{~F}} \cdot \Delta t}{\sqrt{2 \pi} \frac{\tilde{w}_{\text {beam }}}{\left|v_{\mathrm{x}}\right|}} \exp \left(-\frac{\left(t-t_{i}\right)^{2}}{2 \cdot\left(\frac{\tilde{w}_{\text {beam }}}{v_{\mathrm{x}}}\right)^{2}}\right)$.

In practice, the signal $s_{t}\left(t_{1}, t_{2}\right)$ is evaluated (for instance by using an auto-correlation) to directly determine the temporal distance $\left(t_{2}-t_{1}\right)$ of the two scattered light pulses. Together with the previously calibrated beam distance $d_{\text {beam }}$, the flow velocity component $v_{\mathrm{x}}$ perpendicular to the direction of the laser beams is then obtained according to the relation

$v_{\mathrm{x}}=\frac{d_{\text {beam }}}{t_{2}-t_{1}}$.

Sensitivity: The described L2F measurement principle in its original configuration allows to measure a flow velocity component at a single point. The sensitivity direction is perpendicular to the propagation direction of the illuminating laser beams and lies in the same plane as the two laser beams. The observation direction has no effect on the sensitivity direction.

Spatial resolution: The spatial resolution along the sensitivity direction ( $x$-direction) is mainly determined by the beam distance, while the spatial resolution along the beam propagation ( $y$-direction) is determined by the beam divergence or the field of view for the photodetector with respective optics together with the observation angle. The spatial resolution in $z$-direction, i.e., perpendicular to the measurement plane which is defined by the two laser beams, follows from the beam diameter.

Temporal resolution: The temporal resolution for a single particle is the transit time $T_{\mathrm{L} 2 \mathrm{~F}}$. Thus, it depends on the flow velocity and the beam distance. A change of the beam distance will affect the temporal and the spatial resolution. In case of a given measurement time $T$ that is larger than $T_{\mathrm{L} 2 \mathrm{~F}}$, an averaging over floor $\left(T / T_{\mathrm{L} 2 \mathrm{~F}}\right)$ consecutive seeding particles is considered here as the theoretic maximal number of measurements, which is analogous to LDA.

\subsection{PTV/PIV}

The flow is illuminated with two sequential light sheet pulses . The illuminated plane is the measurement plane and the illuminated seeding particles are imaged using an imaging system with a camera, see Fig. 7. Note that spatial variations of the illumination intensity can occur (Nobach 2011), in particular when a Gaussian intensity profile is used for illumination. For the sake of simplicity, a constant illumination intensity profile is subsequently assumed in the observation

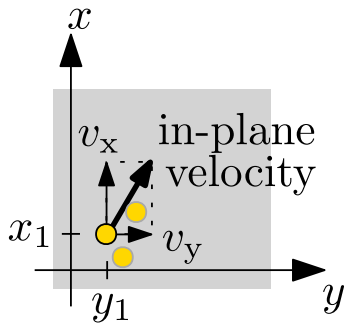

pulse at $t_{1}$

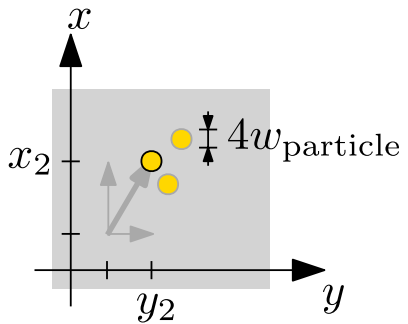

pulse at $t_{2}$
Fig. 7 PTV/PIV measurement principle illustrated with one (PTV) and for instance three (PIV) seeding particles in the interrogation region. The light sheet is shown in side-view, which is the camera view when the observation direction is chosen perpendicular to the light sheet plane

region. Furthermore, the image quantization due to the limited pixel size of the camera is neglected in the present study, because it is considered a technological limitation depending on the available cameras. If the pixel size is near or larger than the size of the particle image and cannot be neglected, the resulting Cramér-Rao bound is higher than in the present study, see (Wernet and Pline 1993) for Poisson noise and (Westerweel 1997, 2000) for Gaussian noise. Assuming a negligibly small pixel size thus leads to the physically lowest possible Cramér-Rao bound and a signal model with a continuous space variable. As a result, each image of a particle in unit number of detected scattered photons (accumulated over the area $\Delta x \cdot \Delta y$ of one pixel) as a function of the inplane coordinates $x$ and $y$ can be approximately described by the two-dimensional Gaussian function

$s_{x, y}\left(x_{i}, y_{i}\right)=\frac{1}{2} \cdot \frac{A_{\mathrm{PTV}} \cdot \Delta x \cdot \Delta y}{2 \pi w_{\text {particle }}^{2}} \exp \left(-\frac{\left(x-x_{i}\right)^{2}+\left(y-y_{i}\right)^{2}}{2 w_{\text {particle }}^{2}}\right)$.

The position $\left(x_{i}, y_{i}\right)$ is the particle position at the time $t_{i}$ of the $i$-th light pulse with $i=1,2$. The $1 / e^{2}$-radius of the particle image amounts to $2 w_{\text {particle }}$. The particle image size in the units of the original space coordinates is either the true particle size or it is increased due to diffraction. The total number of detected scattered photons per seeding particle is $A_{\mathrm{PTV}} / 2$ for each of both images and does not depend on the flow velocity. Although $A_{\mathrm{PTV}}$ can be adjusted directly by varying the pulse energy of the laser, the definition

$A_{\mathrm{PTV}}=\dot{N}_{\text {photon,PTV }} \cdot T_{\mathrm{PTV}}$

with a mean photon rate $\dot{N}_{\text {photon,PTV }}$ over the pulse interval time

$T_{\mathrm{PTV}}=t_{2}-t_{1}$ 
is in principle also possible analogous to a continuous illumination, cf. LDA and L2F.

Note that the task of solving Eq. (14) for the two unknown particle coordinates $x_{i}, y_{i}$ can be transformed in principle to the task of separately solving one equation for each coordinate. By integrating over $x$ or $y$ and multiplying with the respective pixel size $\Delta x$ or $\Delta y$, the separate signals in unit number of photons (accumulated over one sample size) that do solely depend on $x_{i}$ or $y_{i}$, respectively, read

$s_{x}\left(x_{i}\right)=\frac{1}{2} \cdot \frac{A_{\mathrm{PTV}} \cdot \Delta x}{\sqrt{2 \pi} w_{\text {particle }}} \exp \left(-\frac{\left(x-x_{i}\right)^{2}}{2 w_{\text {particle }}^{2}}\right)$,

$s_{y}\left(y_{i}\right)=\frac{1}{2} \cdot \frac{A_{\mathrm{PTV}} \cdot \Delta y}{\sqrt{2 \pi} w_{\text {particle }}} \exp \left(-\frac{\left(y-y_{i}\right)^{2}}{2 w_{\text {particle }}^{2}}\right)$.

The resulting PTV/PIV signal model for the $x$-coordinate is shown in Fig. 8.

In practice, the two sequential particle images according to Eq. (14) are cross-correlated to directly determine the particle position change $\left(x_{2}-x_{1}, y_{2}-y_{1}\right)$ during the interval time $T_{\mathrm{PTV}}$ of the two pulses. Assuming a constant flow velocity, the in-plane velocity components then follow from the calculation

$v_{\mathrm{x}}=\frac{x_{2}-x_{1}}{T_{\mathrm{PTV}}} \quad$ and $\quad v_{\mathrm{y}}=\frac{y_{2}-y_{1}}{T_{\mathrm{PTV}}}$.

Having more than one particle image in the interrogation region, which is the case for PIV in contrast to PTV, the measurement procedure remains the same. However, the resulting velocity measurement is an average over all $N_{\text {particle,PIV }}$ particles in the interrogation region according to the seeding particle concentration.

Sensitivity: The described standard PTV/PIV measurement arrangement has imaging capability and allows to measure simultaneously the axial velocity component and one lateral velocity component with respect to the

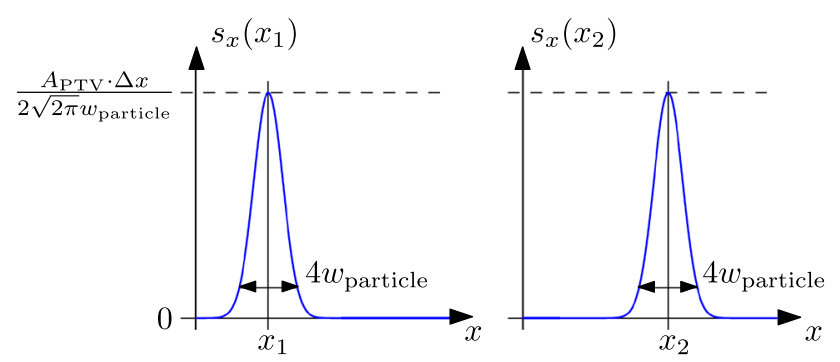

Fig. 8 PTV/PIV signal model over the $x$-coordinate after integrating over the $y$-coordinate illumination. Both sensitivity directions result from the chosen illumination.

Spatial resolution: A perpendicular arrangement between the light sheet plane and the camera direction is a preferred measurement configuration, since it allows to achieve a high spatial resolution. The spatial resolution along the out-ofplane direction is then mostly determined by the light sheet thickness (illumination path) and typically not by the depth of focus of the imaging system (observation path). On the contrary, the spatial resolution along the in-plane directions is usually determined by the chosen interrogation region (observation path) and not by the illumination (PIV). However, the minimal achievable spatial resolution is determined by the particle motion between the two light pulses (PTV).

Temporal resolution: The temporal resolution is given by the pulse interval time $T_{\mathrm{PTV}}$ and is adjustable by varying the temporal distance between both light pulses. Note, however, that the pulse interval time is also connected with the minimal achievable spatial resolution that is the particle motion. If a measurement time $T$ is possible that is larger than a pulse interval time constraint, which follows from a spatial resolution requirement, a theoretic maximum of floor $\left(T / T_{\mathrm{PTV}}\right)$ measurements can be performed. Furthermore, multiple particles in the interrogation region can be evaluated simultaneously for PIV. Note, however, that the respective averaging over multiple measurements according to the derived signal model reflects only the case of non-overlapping particle images, which is the optimal case.

\subsection{Comparison}

A key difference between the different measurement principles is that PDV measures a mixture of in-plane and out-ofplane velocity component (with respect to the measurement plane defined by the illumination), LDA and L2F measure the in-plane velocity component transverse to optical axis of the illumination and PTV/PIV allows to measure both inplane velocity components. Note that this statement holds when only a single illumination direction is allowed. On the contrary, PDV is the only principle where the sensitivity depends on the observation direction. It seems important to remind that a reference $\mathrm{LDA}$, in contrast to the differential LDA that is considered here, actually has the same capability. The reason is that PDV and LDA are based on the Doppler effect, which is affected by the illumination and the observation direction. With the differential LDA setup, which is nowadays more common and therefore considered here, the influence of the observation direction is eliminated by evaluating the difference of two Doppler effects resulting from two different illumination directions but one common observation direction. Due to the different sensitivity direction, the PDV measurement capabilities are not one-to-one 
comparable with the respective capabilities of LDA, L2F and PTV/PIV.

The relevant feature for the velocity measurement with L2F and LDA is a characteristic size of the illumination profile. For L2F, it is the radius of the laser beams, and for LDA, it is the fringe distance. The size of the respective feature is diffraction-limited according to the illumination. The particle size is typically smaller than this feature, but the particle size can limit the feature size if it is larger than the illumination diffraction limit. The opposite is true for PTV/PIV, where the relevant information feature for the velocity measurement is the particle image size, which is a diffraction-limited observation feature. If the particle size is smaller than the diffraction limit, the feature size is limited according to the diffraction limit. For PDV, the relevant information feature is the light wavelength (or the respective mean light frequency), which is a characteristic size in axial direction, i.e., in the light propagation direction. Therefore, both the illumination direction (particle motion with respect to the illumination direction) and the observation direction (particle motion with respect to the observation direction) have an influence on the resulting Doppler effect.

While LDA and L2F optimally operate for a single particle in the interrogation region, PIV and PDV allow measurements with multiple particles in the interrogation region without degrading the sensitivity. In case of PIV, however, the particles should not overlap to not degrade the sensitivity. Therefore, the highest number of simultaneous particle velocity measurements (which means the highest seeding particle concentration) is possible with PDV.

Regarding the spatiotemporal resolution (in the sensitivity direction), the spatial resolution for LDA and L2F is defined by the chosen spatial distribution of the illumination while the temporal resolution then follows according to the seeding particle motion (transit time). For PTV, the temporal illumination characteristic sets the temporal resolution, and the spatial resolution then follows from the respective particle movement. For PDV and PIV, the spatial and the temporal resolutions are defined by the illumination and observation in space and time.

\section{Method of uncertainty propagation that yields the Cramér-Rao bound}

A measurement signal $\tilde{s}_{m}$ in unit number of photons is considered that depends on a single measurand $\theta$ and contains $M$ uncorrelated samples:

$\tilde{s}_{m}=\underbrace{f_{m}(\theta)}_{=s_{m}}+w_{m}, \quad m=1, \ldots, M$.
The symbol $w_{m}$ denotes the signal noise. In order to investigate the shot noise limit, each measurement signal sample is assumed to follow a Poissonian distribution, i.e., the variance of the signal sample $\tilde{s}_{m}$ equals the expectation value: $\operatorname{Var}\left(\tilde{s}_{m}\right)=\mathrm{E}\left(\tilde{s}_{m}\right)$. The expectation value is subsequently denoted by the symbol $s_{m}$, i.e., $\mathrm{E}\left(\tilde{s}_{m}\right)=s_{m}$. Note that a Poissonian photon distribution minimizes Heisenberg's uncertainty relation (Saleh and Teich 2007), so that the considered case is a quantum mechanical lower limit.

According to the estimation theory of Cramér and Rao, the minimal achievable variance for any unbiased estimator $\hat{\theta}$ of the unknown measurand $\theta$ reads

$\operatorname{Var}(\hat{\theta}) \geq \frac{1}{\sum_{m=1}^{M}\left(\frac{\partial s_{m}}{\partial \theta}\right)^{2} \frac{1}{\operatorname{Var}\left(\tilde{s}_{m}\right)}}$

for the given signal in Eq. (20) with uncorrelated noise (Casella and Berger 1990). The right side of the equation is the Cramér-Rao bound, which is identical to the minimal achievable measurement uncertainty squared (Fischer and Czarske 2014). Note that the Cramér-Rao bound is the information theoretic limit of uncertainty since it follows from the inverse of the Fisher information.

In order to derive Eq. (21) using the uncertainty propagation calculation, the weighted average over the estimations $\hat{\theta}_{m}$ from each signal sample is considered as the measurement result:

$\hat{\theta}=\frac{\sum_{m=1}^{M} \hat{\theta}_{m} \cdot \frac{1}{\operatorname{Var}\left(\hat{\theta}_{m}\right)}}{\sum_{m=1}^{M} \frac{1}{\operatorname{Var}\left(\hat{\theta}_{m}\right)}}$,

with $\frac{1}{\operatorname{Var}\left(\hat{\theta}_{m}\right)}$ as the optimal weighting factor according to the Gauß-Markow theorem. Since the noise $w_{m}$ of the measurement signal is uncorrelated, the estimators $\hat{\theta}_{m}$ are uncorrelated as well. Therefore, the uncertainty propagation calculation at first yields

$$
\operatorname{Var}(\hat{\theta})=\frac{\sum_{m=1}^{M} \operatorname{Var}\left(\hat{\theta}_{m}\right) \cdot\left(\frac{1}{\operatorname{Var}\left(\hat{\theta}_{m}\right)}\right)^{2}}{\left(\sum_{m=1}^{M} \frac{1}{\operatorname{Var}\left(\hat{\theta}_{m}\right)}\right)^{2}}=\frac{1}{\sum_{m=1}^{M} \frac{1}{\operatorname{Var}\left(\hat{\theta}_{m}\right)}} .
$$

Estimating $\operatorname{Var}\left(\hat{\theta}_{m}\right)$ with a second uncertainty propagation calculation based on Eq. (20), the relation then reads

$\operatorname{Var}(\hat{\theta})=\frac{1}{\sum_{m=1}^{M}\left(\frac{\partial s_{m}}{\partial \theta}\right)^{2} \frac{1}{\operatorname{Var}\left(\tilde{s}_{m}\right)}}$. 
Comparing Eqs. (21) and (24), the uncertainty propagation calculation applied to the optimal estimator according to the Gauß-Markow theorem directly leads to the Cramér-Rao bound.

Since the variance of each signal sample is $\operatorname{Var}\left(\tilde{s}_{m}\right)=\mathrm{E}\left(\tilde{s}_{m}\right)=s_{m}$ according to the Poissonian noise and Eqs. (20), (24) finally becomes

$$
\operatorname{Var}(\hat{\theta})=\frac{1}{\sum_{m=1}^{M}\left(\frac{\partial s_{m}}{\partial \theta}\right)^{2} \frac{1}{s_{m}}}
$$

as the shot noise limit of the measurand $\theta$.

For a sufficiently small sampling period, the signal samples $s_{m}$ can be approximated by a continuous signal (for instance over time or space). Considering the continuous signal $s_{t} \approx s_{m}$ over the variable $t=m \cdot \Delta t$ with the sampling period $\Delta t \rightarrow \mathrm{d} t$, the shot noise limit solution in Eq. (25) is writable in the integral form

$\operatorname{Var}(\hat{\theta})=\frac{1}{\frac{1}{\Delta t} \int_{0}^{T}\left(\frac{\partial s_{t}}{\partial \theta}\right)^{2} \frac{1}{s_{t}} \mathrm{~d} t}$

with the measurement interval $T=M \cdot \Delta t$. Note that $s_{t}$ denotes the number of detected photons accumulated over one sampling period $\Delta t$, so that integrating $s_{t}$ over $t$ and dividing by $\Delta t$ yields the same total number of detected photons as for summing up $s_{m}$ over $m$. The result is interpretable as the quotient of the noise power spectral density $\frac{1}{\frac{1}{\Delta t}}$ for one photon and an accumulated (optimal weighted) sensitivity. Enhancing denominator and numerator of the quotient with $\frac{\frac{1}{T}}{\frac{1}{T} \int_{0}^{T} \frac{1}{s_{t} t} \mathrm{~d}}$ as weighting normalization, the quotient can also be considered as an effective noise power spectral density divided by the average sensitivity with an optimal weighting.

Note that the shot noise limit in Eqs. (25) or (26), respectively, is valid when the measurand is the only unknown quantity. Additional unknown quantities can increase the minimal achievable measurement uncertainty, and the calculation of the Cramér-Rao lower bound needs to be enhanced using covariances and matrix calculus (Casella and Berger 1990; Kay 1993). The increased calculation effort is not necessary here, since all enhanced solutions remain bounded by the shot noise limit derived in Eqs. (25) and (26). Both formulas thus represent the sought-after ultimate lower bound of the minimal achievable measurement uncertainty.

\section{Photon shot noise limit results}

The shot noise limit for each measurement principle is calculated according to Eq. (26) and the respective signal model derived in Sect. 2. Here, the integral is evaluated from minus infinity to plus infinity, which implicitly implies a sufficiently large measurement time, in particular for LDA and L2F. The following discussions are thus always focused on the case of a measurement time that is equal or larger than the particle transit time. The calculations for LDA, L2F and PTV/PIV make use of the integral solution $\int_{-\infty}^{\infty} a \cdot \exp \left(-z^{2} /\left(2 b^{2}\right)\right) \cdot \frac{z^{2}}{b^{2}} \mathrm{~d} z=\sqrt{2 \pi} a b$ and all calculations are straightforward.

For LDA, the signal model from Eq. (1) is applied and the resulting variance of the Doppler frequency is converted to the corresponding variance of the measured flow velocity component by using Eq. (4). Note that a fringe spacing is assumed that is significantly smaller than the width of the Gaussian envelope. The assumption is plausible, and it is further valid for heterodyne LDA setups (Czarske 2006), where the cosine signal has an offset frequency. An enhanced study for LDA without this assumption is presented in Sobolev and Feshenko (2006), but note that Gaussian noise is considered there. The resulting Cramér-Rao bound then only differs by a different pre-factor and a marginal deviation below seven percent.

For PDV, the signal model from Eq. (5) is applied, where both signals are treated as one concatenated signal with double length. The resulting variance of the Doppler frequency is then used together with Eq. (8) to determine the corresponding variance of the measurement flow velocity component. For L2F, the shot noise limit for both pulse occurrence times is determined at first using the signal model from Eq. (12). The resulting shot noise limit of the measurement flow velocity component is then obtained by an uncertainty propagation calculation of the uncorrelated estimations of the two occurrence times on the basis of Eq. (13). For PTV/ PIV, the signal model from Eq. (17) is used, and the calculated variance of the particle position is applied to Eq. (19) to yield the shot noise limit for the measured flow velocity component. As a result, the calculated shot noise limits read in summary

$\operatorname{Var}\left(\hat{v}_{\mathrm{LDA}}\right)=\frac{4}{\pi^{2}} \cdot \frac{1}{A_{\mathrm{LDA}}} \cdot\left(\frac{d_{\text {fringe }}}{T_{\mathrm{LDA}}}\right)^{2}$,

$\operatorname{Var}\left(\hat{v}_{\mathrm{PDV}}\right)=1 \cdot \frac{1}{N_{\mathrm{particle}, \mathrm{PDV}} \cdot \tau \cdot A_{\mathrm{PDV}}} \cdot\left(\frac{\sqrt{2} \lambda /|\vec{o}-\vec{i}|}{\frac{\tau^{\prime}}{\tau}}\right)^{2}$, 
$\operatorname{Var}\left(\hat{v}_{\mathrm{L} 2 \mathrm{~F}}\right)=1 \cdot \frac{1}{A_{\mathrm{L} 2 \mathrm{~F}}} \cdot\left(\frac{2 \tilde{w}_{\text {beam }}}{T_{\mathrm{L} 2 \mathrm{~F}}}\right)^{2}$,

$\operatorname{Var}\left(\hat{v}_{\mathrm{PTV} / \mathrm{PIV}}\right)=1 \cdot \frac{1}{A_{\mathrm{PTV}}} \cdot\left(\frac{2 w_{\text {particle }}}{T_{\mathrm{PTV}}}\right)^{2}$,

where the relations from Eqs. (3), (11) and (16) were applied to demonstrate the similar structure of the shot noise limits.

It seems remarkable that all identified shot noise limits in Eq. (27) have an identical structure although the measurement principles are fundamentally different. The common inverse proportionality to the total number of detected photons $A$ is no surprise, because this is a well-known characteristic feature for Poisson noise. Note that the expression $N \cdot \tau \cdot A_{\mathrm{PDV}}$ for PDV is the total number of detected photons for the signal camera. The numerical pre-factors are one for L2F, PDV and PTV/PIV. The pre-factor $\frac{4}{\pi^{2}}=0.41$ for LDA is also near one, so that no significant difference between the different measurement principles exists with respect to the pre-factor. The chosen definition of the particle transit time with respect to the width of the Gaussian envelope (here: the time $T_{\mathrm{LDA}}$ that corresponds to the full $1 / e^{2}$-width of the Gaussian envelope) and the definition of a characteristic length (here: the fringe distance $d_{\text {fringe }}$ ) are finally responsible for the resulting value of the pre-factor.

All shot noise limits are directly proportional to the square of a characteristic length scale, but the characteristic length scale is different for each measurement principle. It is the fringe distance $d_{\text {fringe }}$ for LDA, the laser wavelength $\lambda$ scaled with the inverse of the normalized sensitivity vector length $|\vec{o}-\vec{i}| / \sqrt{2}$ (normalization is with respect to the typical measurement configuration $\vec{o} \perp \vec{i}$ ) for PDV, the beam radius $2 \tilde{w}_{\text {beam }}$ for $\mathrm{L} 2 \mathrm{~F}$ and the particle image radius $2 w_{\text {particle }}$ for PTV/PIV. Furthermore, the shot noise limits are inversely proportional to the square of a characteristic time scale. For LDA, L2F and PTV/PIV, this time scale is the transit time of the particle to pass the measurement volume, whereas for $\mathrm{PDV}$, it is the derivative $\tau^{\prime}=\frac{\mathrm{d} \tau(f)}{\mathrm{d} f}$ of the spectral filter transmission divided by the spectral transmission $\tau$ both evaluated at the mean frequency of the scattered laser light.

\subsection{Comparison for measuring a single particle}

To determine and compare the shot noise limits for the flow velocity measurement with a single seeding particle, the following common conditions are applied to Eq. (27) for the sake of comparability:

- A single particle is measured, i.e., $N_{\text {particle,PDV }}=1$.

- The observation direction is perpendicular to the illumination direction, so that the scattering angle is the same for each measurement principle. Note that for PDV, this condition $\vec{o} \perp \vec{i}$ means $|\vec{o}-\vec{i}|=\sqrt{2}$.

- All light sources provide the same power on average in the measurement region and the working distance for illumination and observation, respectively, and the numerical aperture is equal. As a result, the mean detected photon rate during the measurement time is the same for each measurement principle. Therefore, the total number of detected photons $A$ are expressed by using Eqs. (2), (6), (10) and (15).

- A comparable spatial resolution (in all three dimensions) and a comparable temporal resolution are considered. However, the temporal resolution is inversely proportional to the flow velocity for a given spatial resolution. To describe this behavior, the particle transit times $T$ are expressed by Eqs. (3), (7), (11) and (16). Note that this implies an infinitely large measurement time if the measured velocity component becomes zero. Since this particular case is not meaningful for a practical measurement, the subsequent discussions are focused on a nonzero flow velocity component to be measured.

The shot noise limits for LDA, PDV, L2F and PTV/PIV then read

$\operatorname{Var}\left(\hat{v}_{\text {LDA }}\right)=\frac{4}{\pi^{2}} \cdot \frac{\left|v_{\mathrm{x}}\right|^{3}}{\dot{N}_{\text {photon,LDA }}} \cdot \frac{\left(d_{\text {fringe }}\right)^{2}}{\left(4 w_{\text {fringes }}\right)^{3}}$,

$\operatorname{Var}\left(\hat{v}_{\mathrm{PDV}}\right)=\frac{|v| \cdot \tau}{\dot{N}_{\text {photon,PDV }}} \cdot \frac{(\sqrt{2} \lambda /|\vec{o}-\vec{i}|)^{2}}{\left(\tau^{\prime}\right)^{2} \cdot w_{\text {transit }}}$,

$\operatorname{Var}\left(\hat{v}_{\text {L2F }}\right)=\frac{\left|v_{\mathrm{x}}\right|^{3}}{\dot{N}_{\text {photon,L2F }}} \cdot \frac{\left(2 \tilde{w}_{\text {beam }}\right)^{2}}{\left(d_{\text {beam }}\right)^{3}}$,

$\operatorname{Var}\left(\hat{v}_{\text {PTV/PIV }}\right)=\frac{\left|v_{\mathrm{x}}\right|^{3}}{\dot{N}_{\text {photon,PTV }}} \cdot \frac{\left(2 w_{\text {particle }}\right)^{2}}{\left(x_{2}-x_{1}\right)^{3}}$.

Note that for LDA, the ratio $4 w_{\text {fringes }} / d_{\text {fringe }}$ is the number of fringes. The LDA shot noise limit is thus inversely proportional to the squared number of fringes, which was also found in earlier studies regarding Gaussian noise (Sobolev and Feshenko 2006).

As a result, each shot noise limit of the measurement uncertainty increases with the flow velocity to be measured. For LDA, L2F and PTV/PIV, the variance lower bound is directly proportional to $\left|v_{\mathrm{x}}\right|^{3}$, i.e., to the measurand to the power of three, whereas for PDV, it is only 
directly proportional to $|v|$ if the slope $\tau^{\prime}$ of the spectral filter transmission and the transmission value $\tau$ can be assumed as constant. The condition $\tau^{\prime}=$ const is indeed a plausible approximation for a large range of the Doppler frequency (as long as the scattered light mean frequency remains at the edge of the spectral filter), while $\tau=$ const holds for PDV setups where the laser frequency is adjusted accordingly.

The direct proportionality to the square of the respective characteristic length scale $d_{\text {fringe }}, \lambda, 2 \tilde{w}_{\text {beam }}$ and $2 w_{\text {particle }}$ is unchanged, while the inverse proportionality to the square of the characteristic time scale only remains for PDV, when the dependency of $\tau^{\prime}$ and $\tau$ with respect to the flow velocity is neglected. The respective spatial resolution now occurs in the denominator to the power of three for LDA, L2F, PTV/PIV and to the power of one for PDV. In the following comparison, an equal spatial resolution $w=4 w_{\text {fringes }}=w_{\text {transit }}=d_{\text {beam }}=x_{2}-x_{1}$ is considered. Note that the different measurement principles are compared for a similar spatial resolution regarding each dimension. Having a radially symmetrical beam profile for LDA, for instance, this means to consider L2F beams that are not circular but elliptical. The beam dimension perpendicular to the sensitivity direction is then larger than the beam dimension in the sensitivity direction. Although such an L2F setup is not a typical setup today, it can be realized using cylinder lenses and is a fair way to compare the potential of the $\mathrm{L} 2 \mathrm{~F}$ measurement principle with the LDA measurement principle for one common spatial resolution (and a comparable data rate). Without this consideration of a fully comparable spatial resolution, $\mathrm{L} 2 \mathrm{~F}$ is known to enable lower measurement uncertainties than LDA due to the higher illumination intensity for the case of a comparable illumination light power (Beversdorff et al. 1997).

All shot noise limits are inversely proportional to the mean photon rate, and an equal mean photon rate $\dot{N}_{\text {photon }}$ is considered in the following comparison. Note that the mean photon rate equality is below the PTV capabilities, because the laser pulse energy can be accumulated over a larger period than the actual measurement time. Thus, the pulsed laser at PTV/PIV in principle allows to achieve a higher mean photon rate over the measurement time than the continuous-wave laser at LDA and L2F. This also holds for PDV when a pulsed laser is used for illumination. Although below theoretical capabilities, the subsequent comparison with an equal photon rate in combination with an equal temporal resolution (measurement time) means a comparison with an equal number of detected photons on average, which is of high interest. The question is: Which measurement principle uses the amount of energy in the most effective way to minimize the achievable measurement uncertainty?

To answer this question, Eq. (28) is evaluated for the parameter values listed in Table 1. A spatial resolution of $w=100 \mu \mathrm{m}$ is considered as an example, so that the temporal resolution depends on the flow velocity and is $w /\left|v_{\mathrm{x}}\right|$ or $w /|v|$, respectively. The mean photon rate is set to $\dot{N}_{\text {photon }}=1 \times 10^{9} \mathrm{~s}^{-1}$, which corresponds for instance to a mean power of $373 \mathrm{pW}$ when the laser wavelength amounts to $532 \mathrm{~nm}$. The mean power value is plausible since it is the detected light power that was scattered on a single particle with micrometer or even sub-micrometer dimension. Thus, $\frac{100,000}{\left|v_{x}\right| /(\mathrm{m} / \mathrm{s})}$ photons are detected in total for the given spatial and temporal resolution in dependence on the flow velocity component $v_{x}$. The assumed typical characteristic length scales are the fringe distance $d_{\text {fringe }}=10 \mu \mathrm{m}$ (LDA), the scaled laser wavelength $\sqrt{2} \lambda /|\vec{o}-\vec{i}|=\lambda=532 \mathrm{~nm}$ (PDV), the beam radius $2 \tilde{w}_{\text {beam }}=10 \mu \mathrm{m}$ (L2F), the particle image radius $2 w_{\text {particle }}=5 \mu \mathrm{m}$ (PTV/PIV). Regarding the spectral filter at PDV, the transmission $\tau=0.5$ and the slope $\tau^{\prime}=2.5 \times 10^{-9} \mathrm{~Hz}^{-1}$ of the transmission curve are applied,
Table 1 Parameter values for the numerical evaluation of Eq. (28) in Fig. 9 and the numerical evaluation of Eq. (31) in Fig. 10

\begin{tabular}{llll}
\hline Principle(s) & Parameter & Symbol & Value \\
\hline For Fig. 9 and Fig. 10 & & & \\
All & Spatial resolution & $w$ & $100 \mu \mathrm{m}$ \\
All & Mean photon rate & $\dot{N}_{\text {photon }}$ & $1 \times 10^{9} \mathrm{~s}^{-1}$ \\
LDA & Fringe distance & $d_{\text {fringe }}$ & $10 \mu \mathrm{m}$ \\
PDV & Laser wavelength & $\lambda$ & $532 \mathrm{~nm}$ \\
PDV & Length of the sensitivity vector & $|\vec{o}-\vec{i}|$ & $\sqrt{2}$ \\
PDV & Transmission of the spectral filter & $\tau$ & 0.5 \\
PDV & Slope of the transmission curve & $\tau^{\prime}$ & $2.5 \times 10^{-9} \mathrm{~Hz}^{-1}$ \\
L2F & Beam radius & $2 \tilde{w}_{\text {beam }}$ & $10 \mu \mathrm{m}$ \\
PTV/PIV & Particle image radius & $2 w_{\text {particle }}$ & $5 \mu \mathrm{m}$ \\
Only for Fig. 10 & & & \\
All & Temporal resolution & $T$ & $1 \mathrm{~ms}$ \\
PDV & Simultaneously measured number of particles & $N_{\text {particle,PDV }}$ & 50 \\
PTV/PIV & Simultaneously measured number of particles & $N_{\text {particle,PIV }}$ & 5 \\
\hline
\end{tabular}




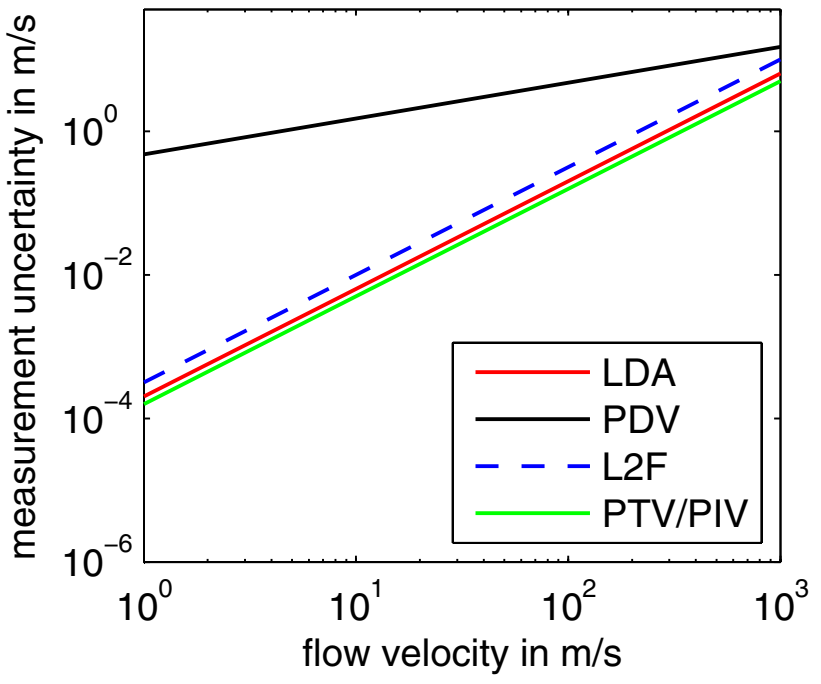

Fig. 9 Calculated measurement uncertainty that is minimal achievable with LDA, PDV, L2F and PTV/PIV due to the shot noise limit as a function of the measurand $v_{\mathrm{x}}$ and $v$, respectively. The calculation is based on the square root of Eq. (28) and is valid for the measurement with one single particle, with a comparable mean photon rate $\dot{N}_{\text {photon }}=1 \times 10^{9} \mathrm{~s}^{-1}$ as well as with a comparable spatial resolution of $w=100 \mu \mathrm{m}$ and temporal resolution $w /\left|v_{\mathrm{x}}\right|$ or $w /|v|$, respectively. A complete list of the applied parameter values is given in Table 1

which are realistic values for a molecular absorption filter filled with iodine gas (Fischer 2017). The finally resulting shot noise limits for LDA, PDV, L2F and PTV/PIV are shown in Fig. 9 as a function of the respective measurand. $\mathrm{L} 2 \mathrm{~F}$ in principle attains the same shot noise limit as PTV/ PIV, but due to the chosen characteristic length scales, the shot noise limit of L2F is here a factor of 2 larger. L2F and LDA have similar shot noise limits, which is considered a remarkable result since the Doppler principle and the time-of-flight measurement principles are complementary principles from a physical perspective. Contrary to the similar shot noise limits of L2F, LDA and PTV/PIV, however, the shot noise limit of PDV is larger for flow velocities below $1.5 \mathrm{~km} / \mathrm{s}$ (L2F), $2.4 \mathrm{~km} / \mathrm{s}$ (LDA) and $3 \mathrm{~km} / \mathrm{s}$ (PTV/PIV), respectively. The sensitivity, which is mainly determined by the slope of the transmission curve of the spectral filter to determine the Doppler frequency, is small in comparison with the LDA light mixing approach. However, a direct comparison of PDV with LDA, L2F and $\mathrm{PTV} / \mathrm{PIV}$ is not possible because PDV measures (at least in parts) the out-of-plane velocity component, whereas the other measurement principles are only sensitive with respect to the in-plane velocity.

Reformulating Eq. (28) by denoting the temporal resolution with the symbol $\Delta T$ and the characteristic length scale for LDA, L2F, PTV/PIV with the symbol $L_{x}$, the uncertainty relation connecting the measurement uncertainty with the temporal resolution reads for LDA, L2F and PTV/PIV

$\operatorname{Var}\left(\hat{v}_{\mathrm{x}}\right) \cdot \Delta T^{3} \sim \frac{L_{\mathrm{x}}^{2}}{N_{\text {photon }}}$

and for PDV

$\operatorname{Var}(\hat{v}) \cdot \Delta T \sim \frac{(\lambda /|\vec{o}-\vec{i}|)^{2}}{\tau \cdot \dot{N}_{\text {photon }}} \cdot\left(\frac{\tau}{\tau^{\prime}}\right)^{2}$.

As a result, an improved temporal resolution means an increase in the velocity measurement uncertainty for a constant mean photon rate and a constant characteristic length. To minimize the uncertainty products on the left side of the formulas, the mean photon rate must be maximized and the characteristic length scale must be minimized. In addition, for PDV, the slope of the filter transmission curve needs to be maximized. Considering the diffraction limit of monochrome light, the characteristic length scale for all measurement principles can be minimized by minimizing the laser wavelength. Furthermore, the characteristic length scale can be minimized by maximizing the numerical aperture of the illumination (LDA, L2F) or observation (PTV/PIV) or between illumination and observation (PDV), which represents a significant difference between the different measurement principles. PTV/PIV can thus cope better than LDA, L2F with large working distances for the illumination, while LDA and L2F seem in principle more suitable to operate with large working distances for the observation.

\subsection{Comparison for measuring multiple particles with averaging}

Two kinds of averaging are possible for the case of measuring on multiple particles. Temporal averaging means that an averaging over consecutive measurements on single particles takes place during a given measurement time $T$. As a maximum, the averaging is possible over $T / T_{\mathrm{LDA}}, T / T_{\mathrm{PDV}}, T / T_{\mathrm{L} 2 \mathrm{~F}}, T / T_{\mathrm{PTV}}$ measurement samples, respectively. In addition, PIV and PDV enable the simultaneous measurement on multiple particles in the interrogation region without disturbance. The latter is true for PIV as long as the particle images do not overlap, whereas no such constraint exists for PDV. Thus, significantly higher seeding particle concentrations can be applied for PDV than for PIV. As a result, an average over $N_{\text {particle,PIV }}<N_{\text {particle,PDV }}$ particles is possible for PIV and PDV.

By applying both kinds of averaging on the shot noise limits from Eq. (28) and using the definitions (3), (7), (11), (16), the shot noise limits for LDA, PDV, L2F and PTV/PIV read 


$$
\begin{aligned}
& \operatorname{Var}\left(\hat{v}_{\text {LDA }}\right)=\frac{4}{\pi^{2}} \cdot \frac{\left|v_{\mathrm{x}}\right|^{2}}{\dot{N}_{\text {photon,LDA }} \cdot T} \cdot\left(\frac{d_{\text {fringe }}}{4 w_{\text {fringes }}}\right)^{2}, \\
& \operatorname{Var}\left(\hat{v}_{\text {PDV }}\right)=\frac{1}{\tau \cdot \dot{N}_{\text {photon,LDA }} \cdot T} \cdot\left(\frac{\sqrt{2} \lambda /|\vec{o}-\vec{i}|}{\tau^{\prime} / \tau}\right)^{2} \cdot \frac{1}{N_{\text {particle,PDV }}}, \\
& \operatorname{Var}\left(\hat{v}_{\text {L2F }}\right)=\frac{\left|v_{\mathrm{x}}\right|^{2}}{\dot{N}_{\text {photon,L2F }} \cdot T} \cdot\left(\frac{2 \tilde{w}_{\text {beam }}}{d_{\text {beam }}}\right)^{2}, \\
& \operatorname{Var}\left(\hat{v}_{\text {PTV/PIV }}\right)=\frac{\left|v_{\mathrm{x}}\right|^{2}}{\dot{N}_{\text {photon,PTV }} \cdot T} \cdot\left(\frac{2 w_{\text {particle }}}{x_{2}-x_{1}}\right)^{2} \cdot \frac{1}{N_{\text {particle,PIV }}} .
\end{aligned}
$$

Remember that the previous calculations are based on the assumption that the measurement time is equal or larger than the particle transit time through the measurement volume. Thus, the derived shot noise limits in Eq. (31) are only applicable for sufficiently large flow velocities. The minimal flow velocity follows from the respective spatial resolution $4 w_{\text {fringes }}, w_{\text {transit }}, d_{\text {beam }}$ or $x_{2}-x_{1}$ divided by the measurement time $T$.

All shot noise limits are inversely proportional to the temporal resolution or the measurement time $T$. Except for PDV, which seems to be velocity-independent, all other shot noise limits now show a proportionality to the flow velocity squared. Therefore, the shot noise limit for LDA, L2F and PTV/PIV can be expressed in the form of a relative measurement uncertainty, whereas it is an absolute measurement uncertainty for PDV. However, note that the PDV result does depend on the velocity, if the linear range of the spectral filter's transmission curve is left and the laser frequency is not adjusted accordingly. Furthermore, all limits are directly proportional to the squared ratio of the characteristic length scale to the spatial resolution.

Inserting the same numerical values from Table 1 already studied in Sect. 4.1 and additionally assuming $N_{\text {particle,PIV }}=5, N_{\text {particle,PDV }}=50$ as an example, the shot noise limits for the measurement time $T=1 \mathrm{~ms}$ are shown in Fig. 10 as a function of the measurand $v_{\mathrm{x}}$ and $v$, respectively. According to the considered spatial resolution and measurement time, the calculation only applies for velocities larger than $0.1 \mathrm{~m} / \mathrm{s}$. Furthermore, a best case scenario is considered for PDV. The laser frequency is always adjusted to measure with the maximal available sensitivity at the edge of the spectral filter's transmission. Therefore, the calculated shot noise limit for PDV is velocity independent.

In comparison with L2F and LDA, the shot noise limit of PTV/PIV is the lowest due to the averaging over simultaneously measured particles. Being independent on the flow

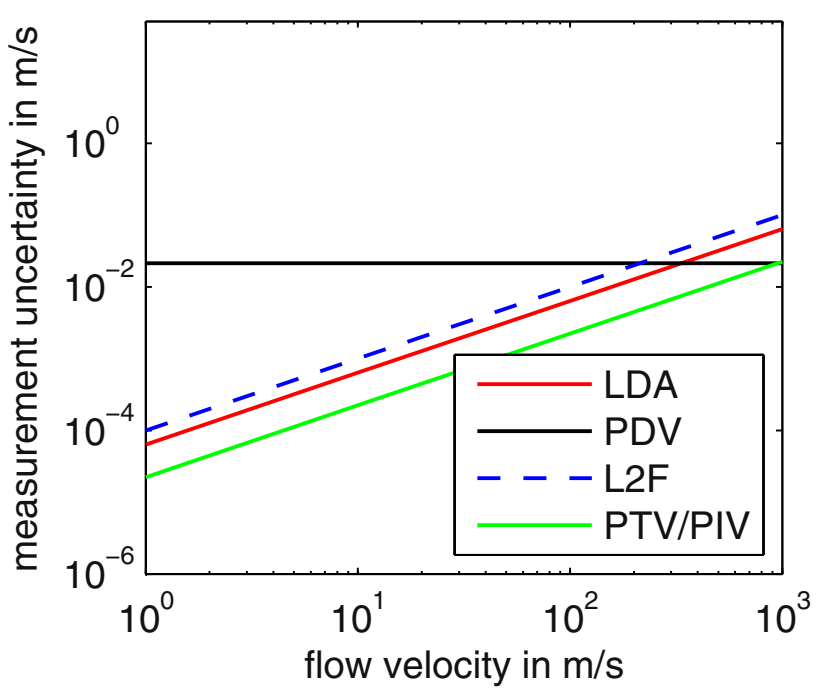

Fig. 10 Calculated measurement uncertainty that is minimal achievable with LDA, PDV, L2F and PTV/PIV due to the shot noise limit as a function of the measurand $v_{\mathrm{x}}$ and $v$, respectively. The calculation is based on the square root of Eq. (31) and is valid for the measurement with multiple particles and averaging. Note that a common mean photon rate $\left(\dot{N}_{\text {photon }}=1 \times 10^{9} \mathrm{~s}^{-1}\right)$ and a comparable spatial resolution $(w=100 \mu \mathrm{m})$ and temporal resolution $(T=1 \mathrm{~ms})$ are considered here. Note further that all parameter values used for the calculation are listed in Table 1

velocity and coping well with an even higher seeding concentration, the shot noise limit of PDV becomes lower than the shot noise limits of L2F, LDA and PTV/PIV at flow velocities $>210 \mathrm{~m} / \mathrm{s},>330 \mathrm{~m} / \mathrm{s}$ and $>950 \mathrm{~m} / \mathrm{s}$, respectively.

Reformulating Eq. (31) by denoting each characteristic length scale with the symbol $L_{\mathrm{x}}$ (LDA, L2F, PTV/PIV) or $\sqrt{2} \lambda /|\vec{o}-\vec{i}|(\mathrm{PDV})$, respectively, the uncertainty relation connecting the measurement uncertainty with the spatial resolution $w_{\mathrm{x}}$ and the temporal resolution $T$ reads for LDA, L2F and PTV/PIV

$\operatorname{Var}\left(\hat{v}_{\mathrm{x}}\right) \cdot T \cdot w_{\mathrm{x}}^{2} \sim\left|v_{\mathrm{x}}\right|^{2} \cdot \frac{L_{\mathrm{x}}^{2}}{\dot{N}_{\text {photon }} \cdot N_{\text {particle }}}$

and for PDV

$\operatorname{Var}(\hat{v}) \cdot T \sim \frac{(\lambda /|\vec{o}-\vec{i}|)^{2}}{\tau \cdot \dot{N}_{\text {photon }} \cdot N_{\text {particle }}} \cdot\left(\frac{\tau}{\tau^{\prime}}\right)^{2}$.

Eq. (33) is identical with Eq. (30) for $N_{\text {particle }}=1$, i.e., the uncertainty relation is the same in both cases, single and multiple particle measurement. Contrary to this, the uncertainty relation for LDA, L2F and PTV/PIV now includes the spatial resolution on the left side of Eq. (32) and also indicates an increasing uncertainty product with an increase in the flow velocity to be measured. 
In order to show that the shot noise limit is in agreement with Heisenberg's uncertainty principle, limitations due to diffraction are now included in the consideration for LDA, L2F and PTV/PIV. The characteristic length scale $L_{\mathrm{x}}$ for LDA results from the fringe distance and for $\mathrm{L} 2 \mathrm{~F}$ from the beam radius. Both are illumination features that are limited in size according to the Gaussian beam characteristics (illumination diffraction limit): $L_{\mathrm{x}} \sim \lambda / \tan \left(\Theta_{\mathrm{x}, \text { illumination }}\right)$ with the opening angle $\Theta_{\mathrm{x} \text {,illumination }}$ of the illumination. For PTV/PIV, $L$ is the particle image size, and because of being an imaging technique the minimal resolvable size follows from Abbe's law (observation diffraction limit): $L_{\mathrm{x}} \sim \lambda / \sin \left(\Theta_{\mathrm{x}, \text { observation }}\right)$ with the opening angle $\Theta_{\mathrm{x}, \text { observation }}$ of the observation. Dividing Eq. (32) by $T \cdot\left|v_{\mathrm{x}}\right|^{2}$ and applying the diffraction limit for $L_{\mathrm{x}}$ in the direction of sensitivity with the approximation $\tan \left(\Theta_{\mathrm{x}, \text { illumination }}\right) \approx \sin \left(\Theta_{\mathrm{x} \text {,illumination }}\right)$ for sufficiently small respective opening angles $\Theta_{\mathrm{x}}$ yields for LDA, L2F and PTV/PIV

$\underbrace{\operatorname{Var}\left(\hat{v}_{\mathrm{x}} \cdot \frac{w_{\mathrm{x}}}{\left|v_{\mathrm{x}}\right|}\right)}_{\delta(x)^{2}} \cdot \underbrace{\left(\frac{\sin \left(\Theta_{\mathrm{x}}\right)}{\lambda}\right)^{2}}_{\sim \delta\left(k_{\mathrm{x}}\right)^{2}} \sim \frac{1}{\dot{N}_{\text {photon }} \cdot T \cdot N_{\text {particle }}}$.

In other words (after calculating the square root): The position uncertainty $\delta(x)$ of the measured particles times the uncertainty $\delta\left(k_{\mathrm{x}}\right)$ of the wave vector component in the direction of sensitivity is inversely proportional to the square root of the total number of detected photons. The uncertainty relation holds for PTV/PIV also for $\delta(y)$ and $\delta\left(k_{\mathrm{y}}\right)$, respectively. The derived result is thus in agreement with Heisenberg's uncertainty principle and other shot noise studies with respect to position measurements (Pavliček and Pech 2016).

Regarding PDV with a molecular absorption filter as spectral filter, the Doppler broadening of the spectral absorption due to the moving absorbing molecules has a Gaussian shape with a full width at half-maximum that is directly proportional to the molecular resonance frequency (Svelto 2010). Furthermore, the resonance frequency is close to the scattered light frequency $f$. Therefore, it is assumed that the maximal slope of the spectral transmission is approximately $\tau^{\prime} \sim 1 / f$ and the working point is at $\tau=0.5$. Inserting both assumptions in Eq. (33), dividing by $T$ and enhancing the expression on the left side by the square of the particle transit time $T_{\mathrm{PDV}}=w_{\text {transit }} /|v|$ (definition see Eq. (7)) results in

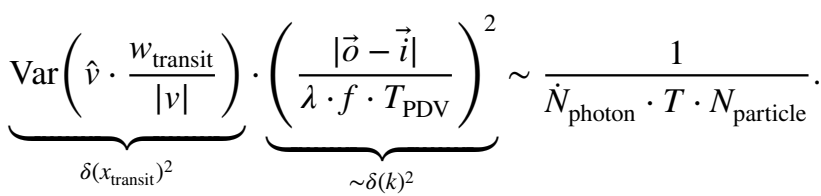

The derived uncertainty principle now indicates a relationship between the position uncertainty $\delta\left(x_{\text {transit }}\right)$ and the uncertainty $\delta(k)$ of the wave vector component in the direction of sensitivity. The latter follows since the inverse of the particle transit time $1 / T_{\mathrm{PDV}}$ represents a resolution limit $\delta(f)$ of the light wave frequency $f$ according to the time-frequency uncertainty principle. Note that this is not a transverse but an axial light property. Furthermore, the relation $\delta(f) \sim c \cdot \delta(k)$ holds due to the wave number definition $k=\frac{2 \pi}{\lambda}$ and $\lambda=c / f$ with the light speed $c$. Since the observed light frequency depends on the difference between illumination and observation direction, a scaling factor of $|\vec{o}-\vec{i}|=2 \sin \frac{\Theta}{2}$ additionally occurs with $\Theta=\angle(\vec{o}, \vec{i})$ as the angle between the observation vector and the illumination vector (McKenzie 1996). As a result, the identified physical uncertainty principle for PDV in Eq. (35) has the same structure as for LDA, L2F and PTV/PIV in Eq. (34). Both identified uncertainty principles obey the same physical law, which is applied to different sensitivity directions.

\section{Conclusion and outlook}

The lower limit of the measurement uncertainty for a signal with a single unknown measurand was derived on the basis of an efficient estimator according to the Gauß-Markow theorem and the well-known rules of uncertainty propagation calculation. Inserting Poissonian noise, the shot noise limit was then provided for discrete signal samples and continuous signals. Note that the restriction to one unknown parameter (the measurand) means that an ultimate lower bound for the minimal achievable measurement uncertainty is obtained.

The general solution to determine the shot noise limit was further applied for LDA, PDV, L2F and PTV/PIV. As a result, the shot noise limits for LDA, L2F and PTV/PIV have an identical structure. PDV is different because it is the only measurement principle that is sensitive with respect to the out-of-plane velocity component. Due to the different sensitivity direction and the different measurand, respectively, a one-to-one comparison with the other measurement principles is difficult. However, a numerical example revealed that the minimal achievable measurement uncertainty due to photon shot noise is comparably large although the largest number of particles can be measured simultaneously. Note that the numerical comparison was performed for an identical spatiotemporal resolution as well as an identical mean photon rate.

The shot noise limits were studied for the case of measuring a single seeding particle and the case of measuring multiple seeding particles and averaging. Averaging multiple particle measurements over a given measurement time leads to a minimal achievable velocity measurement uncertainty that is directly proportional to the absolute value of the velocity (LDA, L2F, PTV/PIV) and that is independent on the velocity value (PDV), respectively. Studying the 
dependency on the spatiotemporal resolution, the measurement uncertainty is always inversely proportional to the square root of the measurement time that here is the temporal resolution. The measurement uncertainty of the flow velocity is further inversely proportional to the spatial resolution for LDA, L2F, PTV/PIV and it does not depend on the spatial resolution for PDV, when an identical mean photon rate is considered.

For all measurement principles, the shot noise limit could be shown to obey an uncertainty principle that is in agreement with Heisenberg's uncertainty principle and Poisson statistics: The product of the position uncertainty (that is the velocity uncertainty times the particle observation time in the interrogation region) and the uncertainty of the respective wave vector component along the sensitivity direction is inversely proportional to the square root of the total number of detected photons. As a result, all studied Doppler and time-of-flight measurement principles obey the same fundamental limit from the perspective of quantum mechanics.

The theoretically identified limits provide a common solid basis to assess different laser-based flow velocity measurement systems with respect to what is feasible from the perspective of photon shot noise (fundamental physical limitation). Experimental studies must follow in the next step, in order to demonstrate shot noise-limited measurements for each measurement principle and to validate the theoretical findings. Furthermore, the dependency of the scattering light power and the signal's sensitivity on the particle size as well as the angular scattering characteristic should be included in the estimation of the minimal achievable measurement uncertainty in future. Finally, shot noise is typically not the only limiting factor for laser-based flow velocity measurements. Another fundamental physical aspect would be to compare the measurement capabilities for a non-constant flow velocity, which means to consider the effect of an accelerated flow and flow turbulence on the flow velocity measurement. Furthermore, the signal processing efficiency should be included in future comparison studies, because the velocity estimation algorithm might not be able to attain the Cramér-Rao bound (signal processing limitation). Future comparison studies should also include the effect of signal quantization over time or space (for PTV see Wernet and Pline 1993 for instance), respectively, as well as other unavoidable error sources such as background light and refractive index changes in flame flows and two- or multi-phase flows. This means to compare the measurement principles with respect to current technological as well as applicationdriven physical limitations.

Acknowledgements The author sincerely thanks the Deutsche Forschungsgemeinschaft (DFG) for funding the research project FI 1989/2-1.
Funding Open Access funding enabled and organized by Projekt DEAL.

Open Access This article is licensed under a Creative Commons Attribution 4.0 International License, which permits use, sharing, adaptation, distribution and reproduction in any medium or format, as long as you give appropriate credit to the original author(s) and the source, provide a link to the Creative Commons licence, and indicate if changes were made. The images or other third party material in this article are included in the article's Creative Commons licence, unless indicated otherwise in a credit line to the material. If material is not included in the article's Creative Commons licence and your intended use is not permitted by statutory regulation or exceeds the permitted use, you will need to obtain permission directly from the copyright holder. To view a copy of this licence, visit http://creativecommons.org/licenses/by/4.0/.

\section{References}

Adrian RJ (1991) Particle-imaging techniques for experimental fluid mechanics. Annu Rev Fluid Mech 23:261-304

Adrian RJ (2005) Twenty years of particle image velocimetry. Exp Fluids 39(2):159-169

Aizu Y, Asakura T (1987) Principles and development of spatial filtering velocimetry. Appl Phys B Lasers Opt 43(4):209-224

Albrecht HE, Damaschke N, Borys M, Tropea C (2003) Laser Doppler and phase Doppler measurement techniques. Springer, Berlin

Belden J, Truscott TT, Axiak MC, Techet AH (2010) Three-dimensional synthetic aperture particle image velocimetry. Meas Sci Technol 21:125403

Beversdorff M, Förster W, Schodl R, Jentink HW (1997) In-flight laser anemometry for aerodynamic investigations on an aircraft. Opt Lasers Eng 27:571-586

Bohren CF, Huffman DR (2004) Absorption and scattering of light by small particles. Wiley, Weinheim

Büttner L, Schlüßler R, Fischer A, Czarske J (2013) Multipoint velocity measurements in flows using a Fabry-Pérot interferometer. Opt Lasers Eng 51(3):325-333

Cadel DR, Lowe KT (2015) Cross-correlation Doppler global velocimetry (CC-DGV). Opt Lasers Eng 71:51-61

Casella G, Berger RL (1990) Statistical inference. Duxbury Press, Belmont

Cenedese A, Cenedese C, Furia F, Marchetti M, Moroni M, Shindler L (2012) 3D particle reconstruction using light field imaging. In: 16th International Symposium on Applications of Laser Techniques to Fluid Mechanics, 1.1.2 (9 pp.). Lisbon

Charrett TOH, Ford HD, Nobes DS, Tatam RP (2004) Two-frequency planar Doppler velocimetry (2-v-PDV). Rev Sci Instrum 75(11):4487-4496

Cierpka C, Segura R, Hain R, Kähler CJ (2010) A simple single camera $3 \mathrm{C} 3 \mathrm{D}$ velocity measurement technique without errors due to depth of correlation and spatial averaging for microfluidics. Meas Sci Technol 17:R71-R91

Coupland J (2000) Coherent detection in Doppler global velocimetry: a simplified method to measure subsonic fluid flow fields. Appl Opt 39(10):1505-1510

Cramér H (1946) A contribution to the theory of statistical estimation. Scandinavian Actuarial Journal 1946(1):85-94

Czarske J (2001) Laser Doppler velocity profile sensor using a chromatic coding. Meas Sci Technol 12:52-57

Czarske JW (2006) Laser Doppler velocimetry using powerful solidstate light sources. Meas Sci Technol 21: 045401 (13 pp.) 
Fahringer TW, Lynch KP, Thurow BS (2015) Volumetric particle image velocimetry with a single plenoptic camera. Meas Sci Technol 26:115201

Fischer A (2016) Fundamental uncertainty limit of optical flow velocimetry according to Heisenberg's uncertainty principle. Appl Opt 55(31):8787-8795

Fischer A (2017) Imaging flow velocimetry with laser Mie scattering. Appl Sci 7(12):1298 (31 pp.),

Fischer A (2017) Model-based review of Doppler global velocimetry techniques with laser frequency modulation. Opt Lasers Eng 93:19-35

Fischer A (2019) Limiting uncertainty relations in laser-based measurements of position and velocity due to quantum shot noise. Entropy 21:264 (19 pp.)

Fischer A, Büttner L, Czarske J, Eggert M, Grosche G, Müller H (2007) Investigation of time-resolved single detector Doppler global velocimetry using sinusoidal laser frequency modulation. Meas Sci Technol 18(8):2529-2545

Fischer A, Czarske J (2014) Measurement uncertainty limit analysis with the Cramér-Rao bound in case of biased estimators. Measurement 54:77-82

Fischer A, Kupsch C, Gürtler J, Czarske J (2015) High-speed light field camera and frequency division multiplexing for fast multi-plane velocity measurements. Opt Express 23(19):24910-24922

Fischer A, Pfister T, Czarske J (2010) Derivation and comparison of fundamental uncertainty limits for laser-two-focus velocimetry, laser Doppler anemometry and Doppler global velocimetry. Measurement 43(10): 1556-1574

Fischer A, Schlüßler R, Haufe D, Czarske J (2014) Lock-in spectroscopy employing a high-speed camera and a micro-scanner for volumetric investigations of unsteady flows. Opt Lett 39(17):5082-5085

Förster W, Karpinsky G, Krain H, Röhle I, Schodl R (2000) 3-Component-Doppler-Laser-Two-Focus velocimetry applied to a transonic centrifugal compressor. In: Proceedings of the 10th International Symposium on Applications of Laser Techniques to Fluid Mechanics, 7.2, pp. 1-12. Lisbon, Portugal

Grosjean N, Graftieaux L, Michard M, Hubner W, Tropea C, Volkert J (1997) Combining LDA and PIV for turbulence measurements in unsteady swirling flows. Meas Sci Technol 8:1523-1535

Heisenberg W (1927) Über den anschaulichen Inhalt der quantentheoretischen Kinematik und Mechanik. Zeitschrift für Physik 43(3): 172-198

Hinsch KD (2002) Holographic particle image velocimetry. Meas Sci Technol 13(7):R61-R72

Hosokawa S, Mastumoto T, Tomiyama A (2013) Tomographic spatial filter velocimetry for three-dimensional measurement of fluid velocity. Exp Fluids 54:1597 (12 pp.)

Jackson DA, Paul DM (1970) Measurement of hypersonic velocities and turbulence by direct spectral analysis of Doppler shifted laser light. Phys Lett 32A(2):77-78

Joint Committee for Guides in Metrology (JCGM): (2008) Evaluation of measurement data - Guide to the expression of uncertainty in measurement. www.bipm.org/en/publications/guides/gum.html

Kay SM (1993) Fundamentals of statistical signal processing. Prentice Hall, London

Lading L, Edwards RV (1993) Laser velocimeters: lower limits to uncertainty. Appl Opt 32(21):3855-3866

Landolt A, Rösgen T (2009) Global Doppler frequency shift detection with near-resonant interferometry. Exp Fluids 47(4-5):733-743

Lu Z.H, Charett T.O.H, Tatam R.P (2009) Three-component planar velocity measurements using Mach-Zehnder interferometric filterbased planar Doppler velocimetry (MZI-PDV). Meas Sci Technol 20(3):034019 (15 pp.)
Maas HG, Gruen A, Papantoniou D (1993) Particle tracking velocimetry in three-dimensional flows-part 1: photogrammetric determination of particle coordinates. Exp Fluids 15(2):133-146

McKenzie RL (1996) Measurement capabilities of planar Doppler velocimetry using pulsed lasers. Appl Opt 35(6):948-964

Meier AH, Rösgen T (2012) Imaging laser Doppler velocimetry. Exp Fluids 52(4):1017-1026

Meyers JF (1995) Development of Doppler global velocimetry as a flow diagnostic tool. Meas Sci Technol 6(6):769-783

Müller H, Eggert M, Czarske J, Büttner L, Fischer A (2007) Singlecamera Doppler global velocimetry based on frequency modulation techniques. Exp Fluids 43(2-3):223-232

Nobach H (2011) Influence of individual variations of particle image intensities on high-resolution PIV. Exp Fluids 50:919-927

Oliver CJ (1980) Accuracy in laser anemometry. J Phys D Appl Phys 13:1145-1159

Pavliček P, Häusler G (2014) Methods for optical shape measurements and their measurement uncertainty. Int J Optomechatronics 8(4):292-303

Pavliček P, Pech M (2016) Shot noise limit of the optical 3D measurement methods for smooth surfaces. Meas Sci Technol 27(3): 035205 (8 pp.)

Rao CR (1945) Information and the accuracy attainable in the estimation of statistical parameters. Bull. Calcutta Math. Soc. 37:81-91

Roehle I, Willert CE (2001) Extension of Doppler global velocimetryto periodic flows. Meas Sci Technol 12:420-431

Saleh BEA, Teich MC (2007) Fundamentals of photonics. Wiley, London

Scarano F (2013) Tomographic PIV: principles and practice. Measur Sci Technol 24: 012001 (28 pp.)

Seiler F, Oertel H (1983) Visualization of velocity fields with Doppler pictures. In: 3rd International symposium on flow visualization. Ann Arbor (Michigan)

Smeets G, George A (1978) Instantaneous laser Doppler velocimeter using a fast wavelength tracking Michelson interferometer. Rev Sci Instrum 49:1589

Sobolev VS, Feshenko AA (2006) Accurate Cramer-Rao bounds for a laser Doppler anemometer. IEEE Trans Instrum Meas 55(2):659-665

Svelto O (2010) Principles of lasers. Springer, Berlin

Tanner L (1973) A particle timing laser velocity meter. Opt Laser Technol 5(3):108-110

Teich MC, Saleh BQA (1989) Squeezed states of light. Quantum Opt J Eur Opt Soc Part B 1(2):153-191

Thacker A, Loyer S, Aubrun S (2010) Comparison of turbulence length scales assessed with three measurementsystems in increasingly complex turbulent flows. Exp Thermal Fluid Sci 34:638-645

Thurow B, Jiang N, Lempert W (2013) Review of ultra-high repetition rate laser diagnostics for fluid dynamic measurements. Meas Sci Technol 24:012002

Voigt A, Bayer C, Shirai K, Büttner L, Czarske J (2008) Laser Doppler field sensor for high resolution flow velocity imaging without camera. Appl Opt 47(27):5028-5040

Wernet MP (2004) Planar particle imaging Doppler velocimetry: a hybrid PIV/DGV technique for three-component velocity measurements. Meas Sci Technol 15:2011-2028

Wernet MP, Pline A (1993) Particle displacement tracking technique and Cramer-Rao lower bound error in centroid estimates from CCD imagery. Exp Fluids 15(4):295-307

Westerweel J (1997) Fundamentals of digital particle image velocimetry. Meas Sci Technol 8:1379-1392

Westerweel J (2000) Theoretical analysis of the measurement precision in particle image velocimetry. Exp Fluids [Suppl.] 29(7):S3-S12 
Willert C, Hassa C, Stockhausen G, Jarius M, Vogesand M, Klinner J (2006) Combined PIV and DGV applied to apressurized gas turbine combustionfacility. Meas Sci Technol 17:1670-1679

Willert C, Stockhausen G, Beversdorff M, Klinner J, Lempereur C, Barricau P, Quest J, Jansen U (2005) Application of Doppler global velocimetry in cryogenic wind tunnels. Exp Fluids 39:420-430
Yeh Y, Cummins HZ (1964) Localized fluid flow measurements with an He-Ne laser spectrometer. Appl Phys Lett 4(10):176-178

Publisher's Note Springer Nature remains neutral with regard to jurisdictional claims in published maps and institutional affiliations. 